\title{
Fairness Evaluation of Practical Spectrum Sharing Techniques in LTE Networks
}

\author{
Francesco Guidolin $^{\dagger} \quad$ Mattia Carpin ${ }^{\dagger} \quad$ Leonardo Badia $^{\dagger *} \quad$ Michele Zorzi $^{\dagger *}$ \\ $\dagger$ Dept. of Information Engineering, University of Padova, via Gradenigo 6/B, 35131 Padova, Italy \\ * Consorzio Ferrara Ricerche, via Saragat 1, blocco B, 44122 Ferrara, Italy
}

\begin{abstract}
In the context of dynamic spectrum access, spectrum sharing among multiple operators has recently emerged as a promising paradigm to improve the efficiency of resource usage. Several theoretical evaluations have proven the benefits offered by pooling the available frequencies so as to tune the capacity offered by the operators according to their different needs, especially the service demands from their users. However, practical aspects concerning the application of sharing techniques are rarely studied, and deserve more detailed investigations. This paper aims at tackling this problem, in particular investigating the impact of asymmetries and dynamics of the user demands on the implementation of spectrum sharing techniques and the resulting performance, especially in terms of fairness among the users, which seems to be often neglected by many studies. We show that in variable traffic conditions, a constantly monitored and updated sharing of frequency bands performs much better than a static allocation simply based on average traffic loads. However, it is possible to choose the update rate of the spectrum allocation so that it does not represent a heavy computational and signaling burden, while retaining most of the improvements brought by the spectrum sharing paradigm.
\end{abstract}

Index Terms-Spectrum sharing, LTE networks, resource management, dynamic spectrum access.

\section{INTRODUCTION}

D URING the last years, due to the deployment of several novel multimedia mobile applications, we witnessed an unprecedented escalation of consumers demand for faster data connectivity. However, the current spectrum usage policies do not permit to extend the bandwidth employed by cellular communication networks. Thus, a more efficient use of the existing spectrum is needed [1].

To match this challenge, new more flexible network setups are appearing. For instance, heterogeneous networks (HetNets) have emerged as one promising configuration of Next Generation Mobile Networks (NGMNs), such as the Long Term Evolution (LTE) of Third Generation cellular networks. While classical cellular networks have a "flat hierarchy," since wireless coverage is guaranteed by one layer of homogeneous Base Stations (BSs), HetNets utilize a higher density of BSs, also with different roles. In particular, "pico" or "femto" base stations (i.e., smaller nodes with reduced power and coverage) can be introduced to locally increase access to the network, even though this may also imply an overlap with existing top-layer BSs and therefore an increase of interference, that has to be properly managed [2]. In this scenario,

This work has been partially supported by the Italian Ministry of University and Education under the bilateral Italy-China project "Coordination techniques for multi-cell cooperative MIMO networks," (no. PGR00195). some techniques for interference management, such as Statical Fractional Frequency Reuse (SFFR), can be employed, but they only mitigate the problem, without solving it. In the end, partitioning the frequency assignment into sub-allocations with exclusive usage may lead to improving the signal to interference plus noise ratio (SINR) perceived by the users, but at the price of decreasing the available bandwidth [3].

Therefore, many researchers advocate the need for an improvement in the radio spectrum usage, reached by sharing, as opposed to simply re-distributing, the available resources. Several papers, projects, and initiatives, have been aimed in the last years at quantifying how theoretical performance limits can be pushed forward by sharing spectrum resources among the operators involved; this leads to estimating the achievable gains from both points of view of technical and also economic performance [4], [5]. We consider here the simplest case, i.e., the so-called orthogonal spectrum sharing, where frequency resources owned by the operators are simply put in common, but their usage is still exclusive by one operator at a time. This can be realized by letting one operator "borrow channels" from another, e.g., when the traffic loads in two neighboring cells are highly asymmetric [6]. Alternatively, this can be originated by the operators pooling all or part of their resources together and defining some sharing policy, for example through a virtual market [7]. We remark that more advanced spectrum sharing policies can be envisioned by making use of Multiple Input Multiple Output (MIMO) techniques, so as to allocate multiple users in the same frequency resource at the same time. Within this scenario, several allocation policies can be adopted to further increase the spectral efficiency, at the cost of a higher complexity [8]. Nevertheless, our investigations in the present paper apply to those cases as well.

When the traffic loads of the operators are known to be asymmetric, there surely is an interest and an immediate gain if both operators share their resources. However, in practice such asymmetries are not precisely known in advance to the operators, and only average values of user demands on a given time-of-day may be known, but not their actual value, which must be estimated in real-time. In this case, it becomes interesting to understand whether the theoretical gain found by spectrum sharing investigations also translates to practical policies that can be implemented in NGMNs. The aim of this paper is to discuss how resources should be shared among the operators according to the traffic load, which may be known either precisely or through a statistical characterization only. We discuss whether an approximate division of the bandwidth 
is still beneficial to achieve spectrum sharing gains, or the number of connected users to each cell should be closely monitored and tracked. To evaluate this, we consider an LTE network modeled in the open-source network simulator 3 (ns3) [9], where we also implemented additional spectrum sharing capabilities not previously available [7]. User arrivals and departures in and from the system are regulated according to memoryless processes with known rates.

Moreover, besides the throughput improvement brought by spectrum sharing, we also investigate fairness among the users. According to our evaluations, getting a precise knowledge of the traffic load asymmetries at a given time instant (as opposed to knowing it only on average) is important to reach a satisfactory fairness among the served users. Thus, each operator should apply spectrum sharing between one of its cells and a neighboring cell belonging to another operator with accurate information about the traffic load of both cells, and even more so if fairness is a key objective for this operator.

These techniques are also discussed in terms of flexibility and computational complexity. It is also shown that a sufficiently precise knowledge of the traffic asymmetries, which can be exploited for spectrum sharing, can be achieved by updating the reported cell loads just every few seconds (or more, depending on the user mobility and call service rate). Therefore, a practical implementation of such techniques can definitely be envisioned as realistic and viable.

The paper is organized as follows. Section II reviews spectrum sharing and details the procedure used in this paper. Section III describes the system model details and the algorithms adopted. Section IV presents the performance of the algorithms evaluated by simulation. Finally, conclusions and future works are presented in Section V.

\section{BACKGROUND ON SPECTRUM SHARING}

Spectrum sharing is a novel paradigm for efficient resource usage in next generation wireless networks [6]. Its motivation is similar to that of cognitive radio, i.e., the overcrowding of available frequencies and the ever-increasing demand for bandwidth from wireless terminals. Also the technological enablers are similar, since both cognitive radio and spectrum sharing involve detection of frequency use, to determine which allocated radio bands are actually underutilized or not even used at all (whitespace detection), and the ability to dynamically access them [10]. Finally, spectrum sharing also often employs cross-layer optimization and applied mathematical tools such as game theory to determine the optimal usage of the resource [11].

However, differently from the most common setup employed in cognitive networks, spectrum sharing does not focus on a primary-secondary interaction (namely, a legitimate radio user who owns the right to transmit/receive on a frequency band, and an opportunistic user that exploits the whitespaces of the primary, respectively), but rather it involves multiple network operators, who all own a licensed spectrum and are willing to cooperate with each other. This can happen for different reasons: from the mathematical standpoint, the motivation is simply the search for a more efficient usage. In

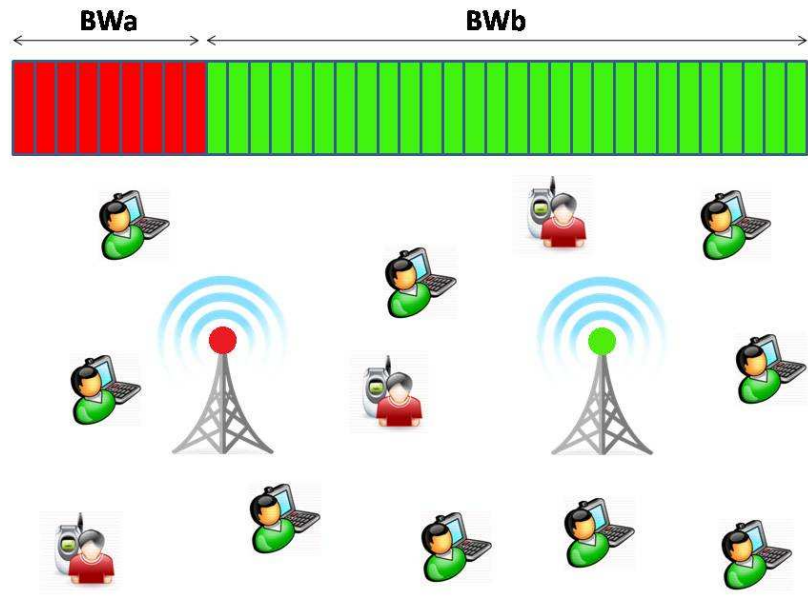

Fig. 1: Two operators managing neighboring cells with spectrum sharing

actual setups involving commercial operators, such a choice can be motivated by joint-venture agreements, mutual rewarding (either monetary or strategical, e.g., in terms of attracting more customers), spectrum leasing operations, and so on [4].

A possible reference scenario is represented in Fig. 1. Here, two neighboring cells are considered, which coexist in the same geographical area but are managed by different operators. The base stations (BSs) of both cells transmit over orthogonal (i.e., disjoint) frequency bands. Therefore, it does not matter whether the cell borders are just adjacent to each other or even overlapping (in principle, the two BSs could even cover the exact same area). Also for the sake of simplicity, in the following we will discuss of resources just seen as "frequencies;" however, in LTE jargon, the resource units to be allocated to the users are resource blocks (RBs), i.e., a combination of time-frequency atomic allocations in orthogonal frequency division multiple access (OFDMA). Precisely, in LTE this corresponds to 12 OFDMA subcarriers allocated for 1 transmission time interval (TTI) that equals $1 \mathrm{~ms}$. Since RBs still represent orthogonal, i.e., non interfering, resources, the reasoning still applies unchanged [12].

Users are spread over the region and are subscribers of either operator. Service to each user is uniquely provided by its serving base station; note that also this assumption is just made for the sake of discussion, but in reality nothing would prevent us from considering a similar setup with BSs possibly handing over users to each other. Spectrum sharing intervenes by determining some of the frequency resources to be re-assigned from their initial owner to the other one, upon necessity.

As a result, the number of users served by each operator varies. This clearly works better whenever asymmetry is present among the traffic loads of the operators; for example, in the figure, the green BS to the right should serve more users than the red BS to the left, thus it makes sense to allocate a larger share of the spectrum to the green operator than to the red one. 
The question we address in this paper is however, how do we quantify that either BS has a "larger" demand than the other? How is "larger" defined, by average values or actual instantaneous values monitored on a close-knit time scale? And most importantly, what is the resulting performance of each of these approaches?

\section{SyStem MODEL}

To answer these questions, we consider users entering the system, requesting service, and leaving upon service completion according to memoryless processes. As a consequence, the number of service requests for each operator during a given time interval is a Poisson distributed random variable, and inter-arrival times $\tau_{1}, \tau_{2}, \ldots, \tau_{n}$ of the users are independent and identically distributed (iid), following an exponential distribution with parameter $\lambda=1 / m_{\tau}$, where $m_{\tau}=\mathbb{E}\left[\tau_{j}\right]$, with $\mathbb{E}[\cdot]$ being the expectation operator. Also, service times $y_{1}, y_{2}, \ldots, y_{n}$ are iid exponential random variables with parameter $\mu=1 / m_{y}$, where $m_{y}=\mathbb{E}\left[y_{j}\right]$.

We denote the two operators as $a$ and $b$, and therefore all the parameters above (i.e., average interarrival and service time, or their reciprocals) may in general have different values for the two operators, which are written as $m_{\tau a}$ and $m_{\tau b}, m_{y a}$ and $m_{y b}$, and so on.

We remark that the choice of a memoryless distribution has been made only for the sake of simplicity in discussing the subsequent numerical results. Indeed, in this case it is possible to describe the asymmetry of the traffic loads, by simply tuning the average inter-arrival and service times; this simplifies the representation of the numerical results. However, we also tried other arrival distributions of the users; in our network simulator, such a choice would be actually fully modular. Therefore, we remark that the evaluations we will show in the following have been qualitatively confirmed also for other kinds of distributions.

Assuming that the bandwidth of each operator is divided in $m \mathrm{RBs}$ and that each operator adopts a Round Robin scheduler, the model can be considered as an $M / M / m$ queue where a user is served immediately if the number of users in service is less than the number of RBs. We consider that this condition is always satisfied, so that the system can be seen as an $M / M / \infty$ queue, thus the number of users in service is equal to the number of users in the system, which is Poisson distributed with mean $m_{x}=\lambda / \mu$ [13]. Actually, any other scenario, e.g., with a different arrival process but the same average number of users, would achieve similar results.

Considering the statistical parameters defined above, we define three different modes for the adaptation of the division of the spectrum among the operators, distinguished as follows.

No Sharing: in this mode, the total available bandwidth is equally divided among the operators without considering the traffic load; this means the absence of spectrum sharing, i.e., each operator just uses the licensed frequencies without "borrowing" anything. This mode is used as a benchmark describing the baseline performance without spectrum sharing. Thus, if $B W_{t o t}$ is the total bandwidth of the system, we have $B W_{a}=B W_{b}=B W_{t o t} / 2$, where $B W_{a}$ and $B W_{b}$ are the portions assigned respectively to the operators $a$ and $b$.
Static mode: the bandwidth is divided proportionally to the average number of users in service. Given that operator $a$ has an average of $m_{x a}$ users in service and operator $b$ has an average of $m_{x b}$ users in service, we obtain

$$
\begin{gathered}
B W_{a}=\left\lfloor B W_{t o t} \frac{m_{x a}}{m_{x a}+m_{x b}}\right\rfloor . \\
B W_{b}=B W_{t o t}-B W_{a}
\end{gathered}
$$

Dynamic mode: the bandwidth is distributed dynamically in each Transmission Time Interval (TTI) proportionally to the number of users in service. If $n_{a}$ and $n_{b}$ are the numbers of users in service during a TTI for operator $a$ and $b$, respectively, we obtain

$$
\begin{gathered}
B W_{a}=\left\lfloor B W_{t o t} \frac{n_{a}}{n_{a}+n_{n b}}\right\rfloor \\
B W_{b}=B W_{t o t}-B W_{a}
\end{gathered}
$$

The aim of this work is to evaluate the performance achievable by the different modes in relation with the statistical parameters described above. We evaluate two metrics of interest: throughput and fairness among the users. The former can be simply quantified through the total transmission rate achieved by the operators. The latter can be evaluated by using Jain's fairness index (denoted as $J$ ) [14]:

$$
J=\frac{\left(\sum_{i=1}^{n} \eta_{i}\right)^{2}}{n \sum_{i=1}^{n} \eta_{i}^{2}}
$$

where $\eta_{i}$ is the throughput of user $i$ and $n$ is the total number of users in the system. We emphasize that $J \in\left[\frac{1}{n}, 1\right]$, where $\frac{1}{n}$ is the value that corresponds to minimum fairness, while 1 indicates perfect fairness among the users.

The last approach presented, i.e., the Dynamic mode, introduces an increase in the implementation complexity by allowing the adaptation of the parameters of the system in real time. In the last part of this work, we study a pseudodynamic approach where the bandwidth is distributed among the operators as in Dynamic mode but the update of the spectrum topology is performed every regular intervals of $T$ seconds starting from an equal division of the spectrum. In particular, we analyze the performance of this algorithm for different values of $T$ for scenarios with different degrees of dynamics.

\section{Performance Evaluation}

To evaluate the performance of the proposed spectrum sharing algorithms in an LTE system, we used the opensource network simulator ns-3.14 [9]. This simulator, based on object oriented programming, includes the entire protocol stack, from the physical to the application layer, and includes a model of LTE technology. Thanks to its modular structure, it has been possible to implement the previously discussed spectrum sharing techniques within an LTE-compliant system, according to the framework reported in [7]. The dynamic spectrum sharing allocation (Dynamic mode) is realized with an instantaneous and genie-like knowledge of the traffic of each operator; this assumption will be relaxed later in this section. 


\begin{tabular}{|c|c|}
\hline Parameter & Value \\
\hline 1-st sub-channel frequency & $2110 \mathrm{MHz}$ \\
\hline Total Downlink Bandwidth & $20 \mathrm{MHz}$ \\
\hline Sub-Carrier Bandwidth & $15 \mathrm{kHz}$ \\
\hline Resource block bandwidth & $180 \mathrm{kHz}$ \\
\hline Resource block carriers & 12 \\
\hline Resource block OFDM symbols & 7 \\
\hline BS downlink TX power & $30 \mathrm{dBm}$ \\
\hline Noise power spectral density & $-174 \mathrm{dBm} / \mathrm{Hz}$ \\
\hline Pathloss at $d$ meters, in dB & $128.1+37.6 \log _{10} d$ \\
\hline Shadow fading & log-normal, zero mean, $\sigma=8 \mathrm{~dB}$ \\
\hline Frame duration & $10 \mathrm{~ms}$ \\
\hline TTI (sub-frame duration) & $1 \mathrm{~ms}$ \\
\hline Target Bit Error Rate & $5 \times 10^{-5}$ \\
\hline Cell radius & $1.5 \mathrm{~km}$ \\
\hline BS distance & $2 \mathrm{~km}$ \\
\hline Simulation Duration & $600 \mathrm{~s}$ \\
\hline
\end{tabular}

TABLE I: Main system parameters

The scenario characteristics follow those reported in Fig. 1 with two BSs, one per operator, positioned at $2 \mathrm{~km}$ of distance. Note that the distance between the BSs does not actually influence the performance, as previously discussed. The mobile users try to receive the same kind of traffic and are uniformly distributed at random in a circular cell with a radius of $1.5 \mathrm{~km}$ centered at the BSs. The total downlink bandwidth available that is distributed between the operators is $20 \mathrm{MHz}$ and is divided into 100 RBs. Moreover, we assume a fully loaded scenario, i.e., the downlink traffic saturates each BS buffer, so all the RBs are used during each frame. Each operator has a total downlink power of $30 \mathrm{dBm}$ that is equally divided among the used RBs. The number of users per BS is variable according to the memoryless process explained in Section III. A comprehensive list of system parameters is reported in Table I. We remark that the simulator includes realistic propagation models and fully LTE-compliant specifications; exhaustive details of the implementation and the parameters used can be found in [7].

In the first simulation, we analyze the evolution of downlink throughput for the two operators in a specific scenario with the following parameters: $m_{\tau a}=10, m_{y a}=80, m_{\tau b}=15$, $m_{y b}=40$. The number of users in service is then unbalanced, since $m_{x a}=8$ and $m_{x b}=2.667$. The results are reported in Figs. 2 and 3. We notice that, as expected, both allocation policies with spectrum sharing (either Static or Dynamic) achieve a higher total throughput, since the operator with more users also gets more resources. However, since the scenario is fully loaded, the throughput improvement is marginal, as it is not related to serving more users, that in any case saturate the available bandwidth, but to a better exploitation of frequency diversity. Operator $a$ can assign resources to a wider set of users and therefore better exploit multi-user diversity by selecting those with better SINR.

The graphs shown above refer to a single scenario, run with the same simulation seeds in the ns-3 simulator. Nevertheless, the trend is exactly the same for any individual scenario.

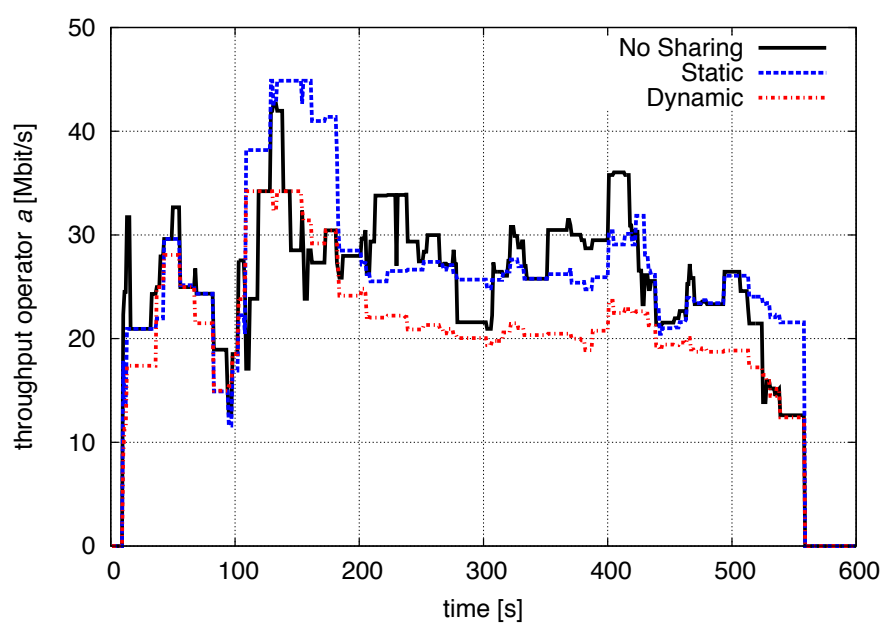

Fig. 2: Throughput of operator $a$.

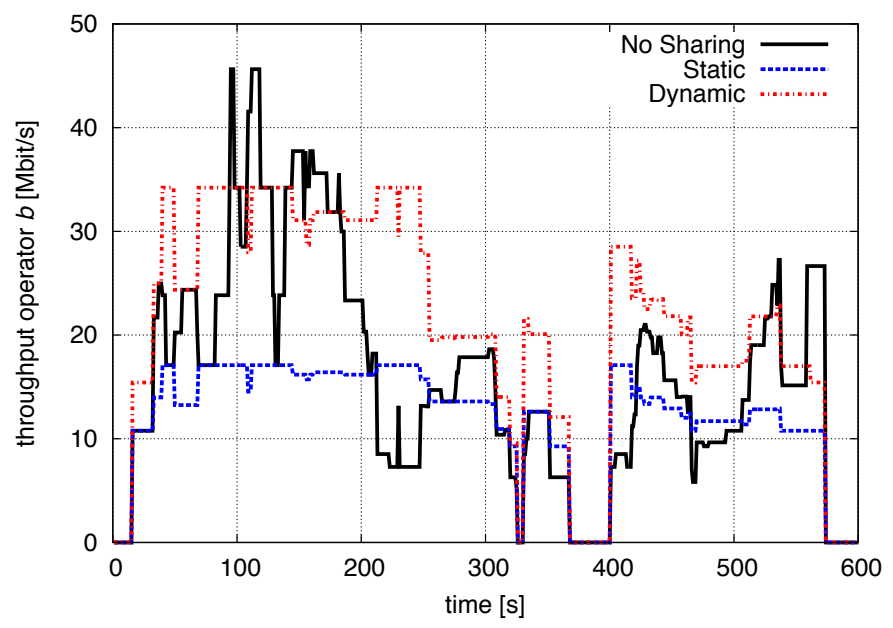

Fig. 3: Throughput of operator $b$.

The general conclusion is that, even though spectrum sharing seems to be better suitable to track load asymmetries of the operators, resulting in some local throughput enhancements, overall the aggregate throughput gain is limited. After all, if the network is properly dimensioned, the entire channel capacity is used.

However, the results also suggest that the usage of resources is significantly improved by spectrum sharing in terms of how they are distributed among the operators, and consequently among the users. For this reason, in the graphs shown in the following, we analyze how the proposed algorithms impact on system fairness, which is in our scenario a more significant metric and is rarely investigated when discussing allocation algorithms for cellular networks, despite being an important characterization of the perceived quality of service. Especially when investigating a game theoretic setup for spectrum sharing, fairness is much easier to frame in the context of mutual relationships among the players.

The following graphs are the results of averaging on a large number of simulation runs (and all the users in the same simulation run) to obtain a statistical confidence close to $99 \%$. 


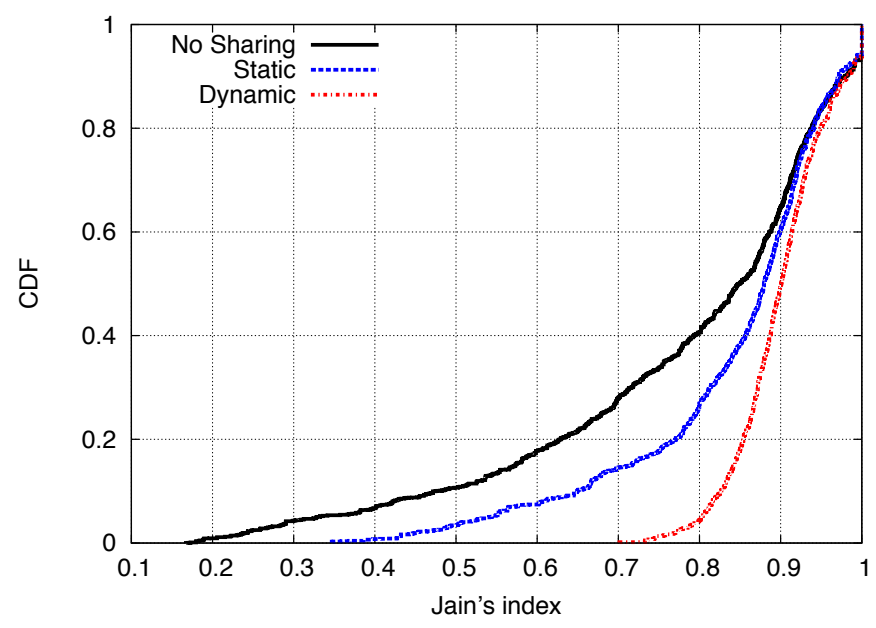

Fig. 4: Jain's index CDF for an unbalanced scenario $(\gamma=3)$.

Firstly, we define a variable that represents the balance degree of the average traffic loads of the operators.

$$
\gamma=\frac{\max \left(m_{x a}, m_{x b}\right)}{\min \left(m_{x a}, m_{x b}\right)}
$$

If $\gamma=1$ the operators are perfectly balanced, while the higher $\gamma$, the lower the balance between the operators.

Fig. 4 describes the cumulative distribution function (CDF) of Jain's index. Here, the curves clearly show that the use of the adaptive spectrum sharing algorithms results in improved fairness, since the resources are more evenly partitioned among the users. Moreover, the flexibility of the Dynamic mode leads to a further improvement, even though at the cost of a slightly higher implementation complexity.

In the second simulation, we evaluate fairness for a scenario with parameters: $m_{\tau a}=10, m_{y a}=40, m_{\tau b}=15, m_{y b}=40$. The number of users in service is more balanced than before, since we obtain $m_{x a}=4$ and $m_{x b}=2.667$. Fig. 5 reports the results. Differently from the previous case, no gain is obtained with the use of a static spectrum sharing policy, which is due to the relatively small difference between the average number of served users. Conversely, a dynamic allocation achieves a better fairness level.

The previous results show how the improvement obtained with the use of the different spectrum sharing algorithms is related to the characteristics of the traffic load at the operators.

Fig. 6 describes the value of the average Jain's index versus the $\gamma$ parameter, i.e., the balance degree defined in (4). By increasing $\gamma$, the fairness performance rapidly decreases for the non-cooperative allocation; when spectrum sharing is employed (Static mode and Dynamic mode), Jain's index remains almost constant. However, a sufficiently high level of fairness (around $J=0.9$ ) is reached only when a dynamic adaptation of the sharing between the two operators is adopted. Compared to a static spectrum sharing, there is a significant fairness gain; notably, this is true for all values of $\gamma$, which means that a dynamic spectrum sharing is able to track also local unbalances of the traffic, even when the operators have a similar average load.

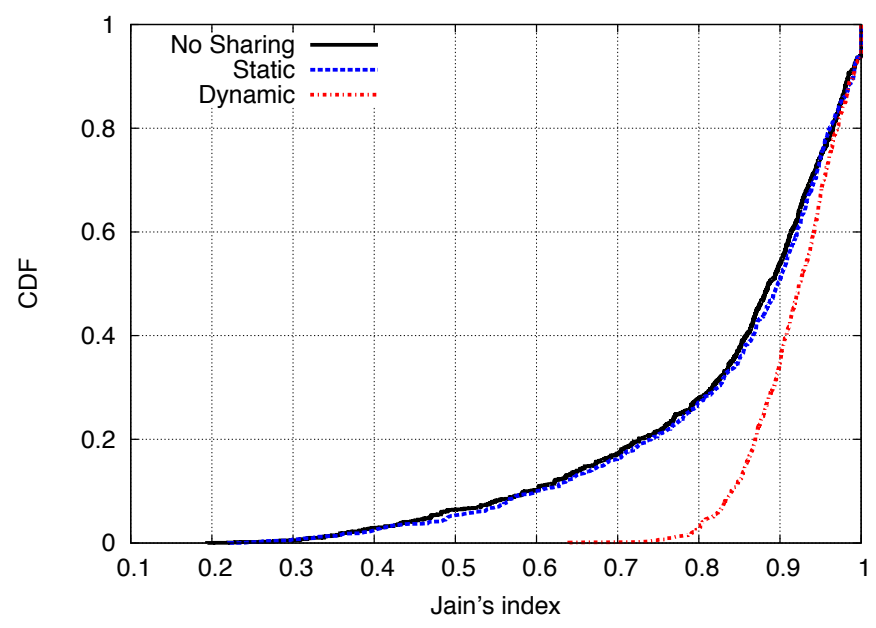

Fig. 5: Jain's index CDF for a more balanced case $(\gamma=1.5)$.

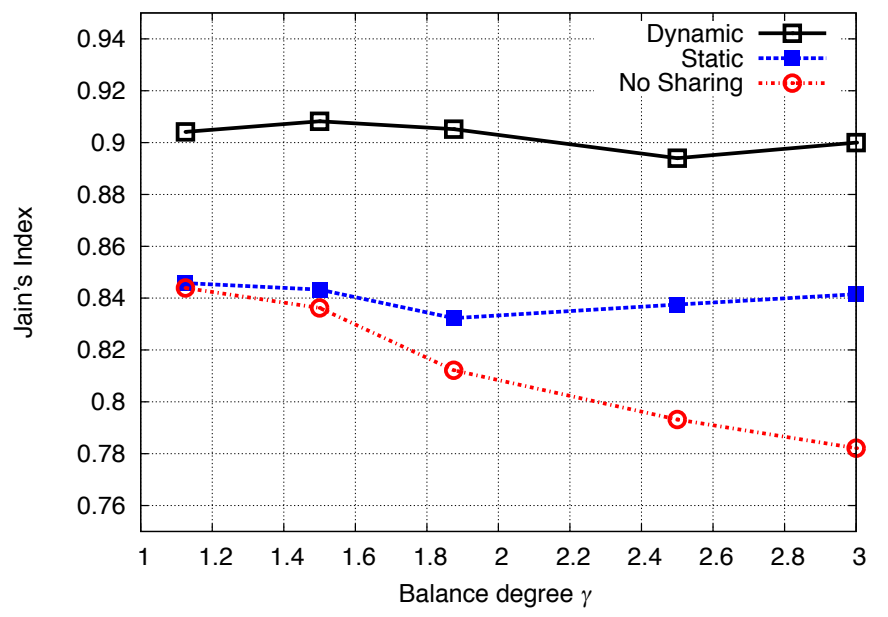

Fig. 6: Average Jain's index as a function of the balance degree of the system.

We can conclude that spectrum sharing is able to offer a better allocation fairness, which translates in a higher quality of service for the users, even when the system is heavily unbalanced [5]. On the other hand, this gain is fully available only if a dynamic spectrum sharing strategy is employed.

Finally, we investigate the flexibility of the Dynamic mode, and the relaxation of the assumption of perfect traffic monitoring of the BSs. In other words, we check how the dynamically updated spectrum sharing allocation, which is shown to achieve the best performance, can deal with the update complexity. Indeed, gaining full knowledge of the user demands for both operators at every time instant would require a considerable overhead burden for the spectrum manager and is likely not feasible in practice.

Thus, we relax the assumption that a dynamic spectrum sharing is realized with genie-like knowledge of the traffic patterns, and instead we consider a possibly outdated information about each operator's traffic, which is periodically updated with a given frequency. This also enables us to evaluate the most proper frequency of update that offers the correct tradeoff between performance and overhead/complexity. We 


\begin{tabular}{|c|c|c|c|c|}
\hline Scenario & $m_{\tau a}$ & $m_{y a}$ & $m_{\tau b}$ & $m_{y b}$ \\
\hline Almost stationary & 20 & 80 & 20 & 60 \\
\hline Slowly variable & 10 & 80 & 15 & 40 \\
\hline Rapidly variable & 2 & 6 & 2 & 4 \\
\hline
\end{tabular}

TABLE II: Scenario parameters

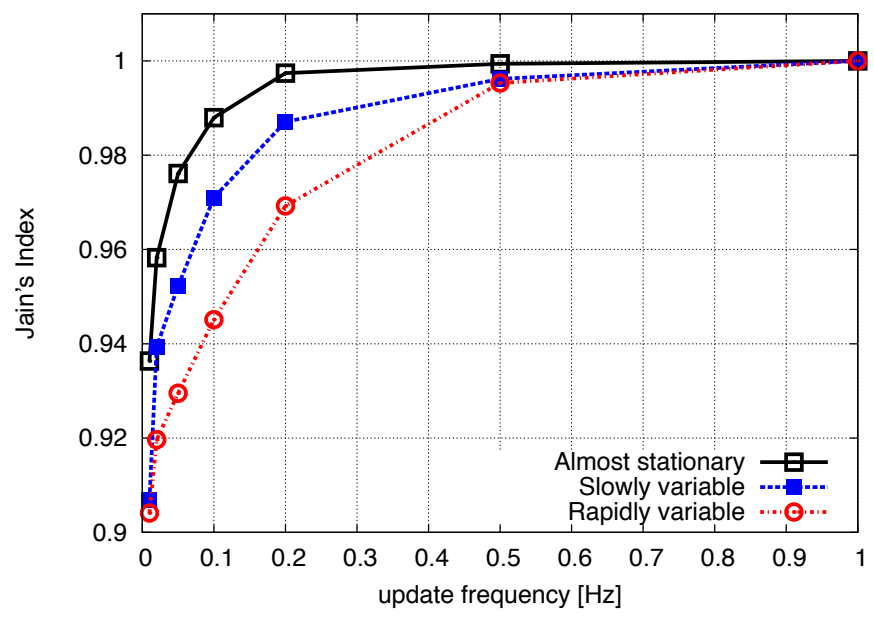

Fig. 7: Average Jain's index as a function of the update frequency for different scenarios.

define three scenarios, with different speed of users' traffic evolution; the difference is in the parameters of the inter-arrival and service times of the users. The parameters of these three scenarios, labeled as "almost stationary," "slowly variable," and "rapidly variable" scenario, are defined in Table II.

We also evaluate the average Jain's index obtained for different update frequencies, i.e., every 1, 2, 5, 10, 20, 50, or 100 s. The results obtained are reported in Fig. 7. We notice that the fairness performance of the different scenarios is almost optimal for update frequencies between 1 and $0.5 \mathrm{~Hz}$. Below $0.5 \mathrm{~Hz}$ we obtain a rapid degradation of the performance in the high dynamics scenario, while for the low dynamics scenario the performance decreases significantly only below $0.05 \mathrm{~Hz}$. Thus, the knowledge of the statistical parameters of the system is important to properly set the update frequency of the spectrum configuration used. In particular, by adopting a frequency update value equal to $1 / \min \left(m_{\tau a}, m_{\tau b}\right)$ it is possible to reach an average Jain's index value corresponding to 0.98 , thereby achieving a good tradeoff between complexity and performance. We emphasize that, since the scenario is fully loaded, the total throughput is not affected by the update frequency.

\section{CONCLUSIONS AND FUtURE WORK}

In this work, we investigated the implementation of spectrum sharing techniques by analyzing different practical aspects, namely, whether the spectrum allocation should track the traffic variations of the operators and how often, and how this impacts the performance, quantified in terms of both throughput and fairness. To validate our findings, we used an open source network simulator, in which we included several extensions, freely available as described in [7], to implement spectrum sharing with adjustable shares for each operator.

We obtained that spectrum sharing is generally beneficial for the system performance, especially when the traffic loads of the operators are heavily unbalanced. Specifically, a significant gain in terms of user fairness is achieved, while total throughput improvements, albeit present, are marginal if the system operates in saturation.

However, these gains require a dynamic approach that updates the spectrum allocation according to the users' demand, while just using average values does not always lead to comparable gains. Furthermore, we also tested how often this dynamic update should be performed, and the main conclusion is that an update period of the order of a few seconds is sufficient to retain the full performance of the approach, and even the degradation by just updating with a period of tens of seconds can be acceptable, depending on how fast the scenario changes. Thus, the associated overhead to a dynamic monitoring is likely to be relatively low and can be beneficial to the whole spectrum sharing procedure.

For future work, we will expand these investigations to more complex scenarios, possibly including different kinds of BSs in a HetNet setup, to see if further practical aspects of spectrum sharing implementation come into play. Moreover, different traffic models, also non-saturated, and more advanced schedulers (e.g., prioritizing between different kinds of traffic) could be considered.

\section{REFERENCES}

[1] Cisco, "Global Mobile Data Traffic Forecast Update, 2011-2016," Feb. 2012, White Paper, pp. 1-29.

[2] J. G. Andrews, "Seven ways that HetNets are a cellular paradigm shift," IEEE Comm. Mag., vol. 51, no. 3, pp. 136-144, Mar. 2013.

[3] S.-E. Elayoubi, O. Ben Haddada, and B. Fouresti, "Performance evaluation of frequency planning schemes in OFDMA-based networks," IEEE Trans. Wirel. Commun., pp. 1623-1633, May 2008.

[4] E. A. Jorswieck, L. Badia, T. Fahldieck, E. Karipidis, and Jian Luo, "Spectrum sharing improves the network efficiency for cellular operators," IEEE Comm. Mag., vol. 52, no. 3, pp. 129-136, Mar. 2014.

[5] G. Middleton, K. Hooli, A. Tolli, and J. Lilleberg, "Inter-operator spectrum sharing in a broadband cellular network," Proc. IEEE ISSSTA, pp. 376-380, Aug. 2006

[6] L. Anchora, L. Badia, E. Karipidis, and M. Zorzi, "Capacity gains due to orthogonal spectrum sharing in multi-operator LTE cellular networks," Proc. IEEE ISWCS, pp. 286-290, Aug. 2012.

[7] L. Anchora, M. Mezzavilla, L. Badia, and M. Zorzi, "A performance evaluation tool for spectrum sharing in multi-operator LTE networks," Elsevier Computer Communications, Nov. 2012.

[8] F. Guidolin, A. Orsino, L. Badia, and M. Zorzi, "Statistical analysis of non orthogonal spectrum sharing and scheduling strategies in next generation mobile networks," Proc. IEEE IWCMC, pp. 680-685, Jul. 2013.

[9] ns-3 simulator. Available: http://www.nsnam.org/.

[10] S. Geirhofer, L. Tong, and B. M. Sadler, "Dynamic spectrum access in the time domain: modeling and exploiting white space," IEEE Comm. Mag., vol. 45, no. 5, pp. 66-72, May 2007.

[11] J. E. Suris, L. A. DaSilva, Zhu Han, and A. B. MacKenzie, "Cooperative game theory for distributed spectrum sharing," IEEE ICC 2007, pp. 5282-5287, 24-28 June 2007.

[12] S. Sesia, I. Toufik, and M. Baker, LTE - The UMTS Long Term Evolution: From Theory to Practice, John Wiley \& Sons, Ltd, Chichester, UK.

[13] L. Kleinrock, Queueing systems, Vol. 1. Theory. John Wiley \& Sons, Inc., New York, NY, 1975.

[14] R. K. Jain, Dah-Ming Chiu, and W. Hawe, "A quantitative measure of fairness and discrimination for resource allocation in shared computer system,” Technical Report TR-301, DEC Research Report, Sept. 1984. 